\title{
INFLUÊNCIA DE DIFERENTES DOSES DE FÓSFORO NO CRESCIMENTO DE MUDAS DE CUMARU
}

\author{
Gisele de Aguiar Lima ${ }^{1}$; Bianca Diniz da Rocha²; Jobert Silva da Rocha ${ }^{3}$; Fabisson Rennan Nunes \\ Alves"; Douglas valente de Oliveira5; Laura Fernanda de Lima Lobato6; Elizandra Patrícia de \\ Oliveira Figueira ${ }^{7}$; Kamila Stephanie Souza Barbosa ${ }^{8}$.
}

\footnotetext{
1Universidade Federal do Oeste do Pará, Santarém, Pará, Brasil, gisele_megan@hotmail.com ¿Universidade Federal do Oeste do Pará, Santarém, Pará, Brasil, bianca_dinizdarocha@hotmail.com

3Universidade Federal do Oeste do Pará, Santarém, Pará, Brasil, jobert.job.rocha@gmail.com

${ }^{4}$ Universidade Federal do Oeste do Pará, Santarém, Pará, Brasil, fabissonunes@gmail.com 5Universidade Federal do Oeste do Pará, Santarém, Pará, Brasil, douglasvalentedeoliveira@hotmail.com

6Universidade Federal do Oeste do Pará, Santarém, Pará, Brasil, laura.fll@hotmail.com

7 Universidade Federal do Oeste do Pará, Santarém, Pará, Brasil, elizandrafigueira@hotmail.com

${ }^{8}$ Universidade Federal do Oeste do Pará, Santarém, Pará, Brasil, kamilastephanie7@gmail.com
}

RESUMO: Este trabalho teve como objetivo avaliar o efeito de diferentes dosagens de fósforo no crescimento de mudas de Cumaru (Dipteryx odorata (Aubl.) Willd.) em condições de viveiro. O experimento foi conduzido em Viveiro experimental da Universidade Federal do Oeste do Pará -UFOPA, no período de Junho a Setembro de 2016. O delineamento experimental utilizado foi o inteiramente casualizado, com 5 tratamentos e 4 repetições, totalizando 20 parcelas. Os tratamentos testados foram: testemunha (sem adição de fósforo), 100, 200, 400, $800 \mathrm{~kg} / \mathrm{ha}$ de fosfato simples. Foi avaliado o crescimento em altura, diâmetro do colo, massa fresca aérea, massa fresca radicular, massa fresca total, massa seca aérea, massa seca radicular, massa seca total e, posteriormente, Índice de Qualidade de Dickson. Seguiram os testes de Kolmogorov-Smirnbov para normalidade dos dados, Bartlett para homogeneidade das variâncias dos tratamentos e Fischer para Análise de Variância e as médias comparadas pelo teste de Tukey a $5 \%$ de probabilidade. Conclui-se que a dosagem de $200 \mathrm{Kg} / \mathrm{ha}$ de P é o tratamento mais significativo ao desenvolvimento de mudas em viveiro. Quanto aos parâmetros de desenvolvimento morfológico das plântulas, apresentaram-se não significativo a todos os tratamentos à base de adubação fosfatada adotados. O Índice de qualidade de mudas calculado (IQD), indicou que todos os níveis de adubação fosfatada testados, geraram mudas aptas a instalação em campo, após 60 dias em condições de viveiro.

PALAVRAS-CHAVE: Dipteryx odorata, Fisiologia vegetal, Nutrição mineral. 


\title{
INFLUENCE OF DIFFERENT DOSES OF PHOSPHORUS IN THE GROWTH OF CUMARU SEEDLINGS
}

\begin{abstract}
This work has the objective of evaluating the effect of different phosphorus dosages in the growth of Cumaru (Dipteryx odorata (Aubl.) Willd.) seedlings in nursery conditions. The experiment was conducted in experimental nursery at the Universidade Federal do Oeste do Pará-UFOPA in the period from June to September 2016. The experimental delineation used was entirely ignited, with 5 treatments and 4 repetitions, totaling 20 parcels. The test treatments were made: witness (no addition of phosphorus), $100,200,400,800 \mathrm{~kg} / \mathrm{ha}$ of simple phosphate. The growth in height, colt diameter, fresh air mass, root mass, total fresh mass, dry drought mass, root drought mass, total drought mass and, subsequently, Dickson Quality Index were evaluated. They followed the Kolmogorov-Smirnbov tests for data normality, Bartlett for homogeneity of the variations of the treatments and Fischer for Analysis of Variance and the medias compared to Tukey's test at 5\% probability. It is concluded that the dosage of $200 \mathrm{Kg} / \mathrm{ha}$ of $\mathrm{P}$ is the most significant treatment for the development in nursery. As regards the parameters of the morphological development of the plates, they appeared not significant to all treatments based on phosphate fertilization. The calculated seedling quality index (IQD) indicated that all levels of phosphate fertilizer tested, weighed seedlings suitable for field installation, after 60 days in nursery conditions.
\end{abstract}

KEYWORDS: Dipteryx odorata, Mineral nutrition, Vegetal physiology.

\section{INFLUENCIA DE DIFERENTES DOSIS DE FÓSFORO EN EL CRECIMIENTO DE CAMBIOS DE CUMARU}

RESUMEN: Este trabajo tuvo como objetivo evaluar el efecto de diferentes dosis de fósforo en el crecimiento de mudas de Cumaru (Dipteryx odorata (Aubl.) Willd.) en condiciones de vivero. El experimento fue conducido en Vivero experimental de la Universidad Federal del Oeste del Pará -UFOPA, en el período de junio a septiembre de 2016. El delineamiento experimental utilizado fue el completamente casualizado, con 5 tratamientos y 4 repeticiones, totalizando 20 parcelas. Los tratamientos probados fueron: testigo (sin adición de fósforo), 100, 200,400, $800 \mathrm{~kg} /$ ha de fosfato simple. Se evaluó el crecimiento en altura, diámetro del cuello, masa fresca aérea, masa fresca radicular, masa fresca total, masa seca aérea, masa seca radicular, masa seca total y, posteriormente, índice de calidad de Dickson. Se siguieron las pruebas de Kolmogorov-Smirnbov para la normalidad de los datos, Bartlett para homogeneidad de las varianzas de los tratamientos y Fischer para Análisis de Varianza y las medias comparadas por el test de Tukey al 5\% de probabilidad. Se concluye que la dosificación de $200 \mathrm{Kg} /$ ha de P es el tratamiento más significativo al desarrollo de las mudas en vivero. En cuanto a los parámetros de desarrollo morfológico de las plántulas, se presentaron no significativos a todos los

Agroecossistemas, v. 10, n. 2, p. $136-146$, 2018, ISSN online 2318-0188 
tratamientos a base de fertilización fosfatada adoptados. El índice de calidad de mudas calculado (IQD), indicó que todos los niveles de fertilización fosfatada probados generaron mudas aptas para la instalación en campo, después de 60 días en condiciones de vivero.

PALABRAS CLAVE: Dipteryx odorata, Fisiología vegetal, Nutrición mineral.

\section{INTRODUÇÃO}

O maior valor comercial do cumaru (Dipteryx odorata (Aubl.) Willd.) está na madeira que é muito pesada (densidade $1,09 \mathrm{~g} / \mathrm{cm} 3$ ) dura ao corte, de textura fina e média, de resistência mecânica elevada e muito resistente ao ataque de cupins e de fungos apodrecedores, no qual é indicada para construção civil, como vigas, caibros, ripas, tábuas e tacos para assoalhos, batentes de portas, lambris, forros, 21 para uso externo, como postes, esteios, estacas, cruzetas, dormentes, para confecção de carrocerias, vagões, defensas, para construção naval, bem como para a fabricação de móveis (LORENZI, 1998).

As florestas no Brasil têm sido implantadas em solos onde normalmente o fósforo é um dos nutrientes mais limitantes ao crescimento do vegetal, onde a produção de mudas, muitas vezes, é feita utilizando-se substratos comerciais ou subsolo, cuja fertilidade é baixa ou desiquilibrado nutricionalmente, outro fator que influência o manejo inadequado é que informações sobre exigências nutricionais de espécies florestais, em especial das essências nativas, são escassas (CECONI et al., 2006).

Além disso, boa parte do fósforo fornecido na adubação é indisponibilizada, em virtude do fenômeno da fixação do P em reações com componentes do solo (REZENDE; NETO, 2007). Isso faz com que a aplicação de quantidades altas de fósforo seja necessária para viabilizar a exploração agrícola e florestal.

Moreira et al. (1991), em seu estudo sobre o efeito do tempo de contato do fósforo com amostras de três solos em mudas de Eucalyptus grandis, concluíram que a disponibilidade de $\mathrm{P}$ para o crescimento das mudas diminuiu 
com o aumento do tempo de contato do fertilizante fosfatado com o solo.

O fósforo é um elemento essencial no metabolismo nas plantas, desempenhando papel fundamental importante na transferência de energia da célula, na respiração e fotossíntese, além de ser componente estrutural dos ácidos nucléicos de cromossomos, assim como de muitas coenzimas, fosfoproteínas e fosfolipídios (ZUCAREL, 2006). Desse modo, limitações relacionadas à limitação deste macronutriente no início do seu ciclo de vida acarreta em restrições no seu desenvolvimento, mesmo com o aumento dos níveis de P de forma adequada.

Diante disso, esse trabalho teve como objetivo avaliar o efeito de diferentes dosagens de fósforo no crescimento de mudas de Cumaru e as suas respostas morfológicas em condições de viveiro.

\section{MATERIAL E MÉTODOS}

O experimento foi conduzido em viveiro experimental de produção de mudas da Universidade Federal do
Oeste do Pará - UFOPA (2²5' 9.528" S e $54^{\circ} 44^{\prime} 30.805^{\prime \prime}$ W), no período de Junho a setembro de 2016. O substrato utilizado foi $60 \%$ de terra preta, $20 \%$ palha de arroz carbonizada e 20\% de serragem curtida.

O delineamento experimental utilizado foi inteiramente casualizado, com cinco tratamentos e quatro repetições, totalizando vinte parcelas. Os tratamentos testados foram: testemunha (sem adição de fósforo), 100, 200, 400, $800 \mathrm{~kg} / \mathrm{ha}$ de fosfato simples, sendo a unidade experimental representada por sacos de polietileno de cinco litros contendo a plântula. Seguiram quatro avaliações quinzenas, no qual era contabilizado o número de folhas (NF), diâmetro do coleto (DC), altura (h) e área foliar (AF) através do comprimento e largura dos folíolos.

Para quantificação do número de folhas, foram consideras apenas as folhas ativas. $O$ diâmetro do caule foi medido através de um paquímetro; a altura foi medida com uma régua graduada em centímetros $(\mathrm{cm})$. A área foliar foi determinada por meio de 
regressão linear $y=79,144 X+795,65$, no qual a variável dependente é o produto do comprimento e largura dos folíolos.

As plantas foram cortadas à altura do colo, e a parte aérea e radicular foi separada. Cada componente foi imediatamente pesada em uma balança analítica de precisão, para determinação do peso da matéria úmida, posteriormente foram acondicionadas em sacos de papel e colocadas para secar em estufa na temperatura de $100^{\circ} \mathrm{C}$, por $48 \mathrm{~h}$, onde posteriormente foi feito a determinação da matéria seca. $\bigcirc$ peso da matéria seca e úmida total foi obtido somando as duas partes.

As fórmulas utilizadas para obter as diversas taxas de crescimento da planta, com base na produção de matéria seca e na área foliar, possibilitando analisar o crescimento, foram as seguintes:

$$
\begin{gathered}
T C A=\frac{P^{2}-P^{1}}{t^{2}-t^{1}} \\
T C R=\frac{\operatorname{Ln} P_{2}-\operatorname{Ln} P_{1}}{t_{2}-t_{1}} \\
T A L=\frac{P_{2}-P_{1}}{t_{2}-t_{1}} \cdot \frac{\operatorname{Ln} A_{2}-\operatorname{Ln} A_{1}}{A_{2}-A_{1}} \\
A F E=A F-M S F
\end{gathered}
$$

Onde TCA é a taxa de crescimento absoluto; TCR é a taxa de crescimento relativo; TAL é a taxa de assimilação líquida; AFE é a área foliar específica.
As análises das mudas iniciaram a partir da instalação do experimento, avaliando-se, inicialmente, crescimento em altura, diâmetro do colo, massa fresca aérea, massa fresca radicular, massa fresca total, massa seca aérea, massa seca radicular, massa seca total e, posteriormente, Índice de Qualidade de Dickson.

O acompanhamento do crescimento foi realizado em um intervalo de 15 em 15 dias através da medição da altura, com régua graduada em centímetros. Para a medição de diâmetro foi necessário a utilização de um paquímetro digital com precisão de 0,01 mm. As medições de altura foram realizadas quinzenalmente, totalizando quatro avaliações.

Ao final do experimento (60 dias), aplicou-se análise pelo método destrutivo das amostras para a avaliação de acúmulo de biomassa. Foi obtido o peso da massa verde da parte aérea, radicular e total, com o uso de uma balança analítica. Posteriormente, para obtenção da massa seca, cada amostra foi levada a estufa incubadora a $100{ }^{\circ} \mathrm{C}$ 
por $24 \mathrm{~h}$ até a secagem total e obtenção de peso constante.

Para calcular O IQD no presente experimento, os parâmetros morfológicos altura (h), diâmetro do coleto (DC), massa seca da parte aérea (MSA), massa seca da parte radicular (MSR) e massa seca total (MST) das plantas foram utilizados, conforme explicito na seguinte equação:

$$
I Q D=\frac{M S T(g)}{\frac{h(\mathrm{~cm})}{D C(\mathrm{~mm})}+\frac{M S A(g)}{M S R(g)}}
$$

Os dados obtidos foram organizados em planilha eletrônica e os testes de Kolmogorov-Smirnbov para normalidade dos dados, Bartlett para homogeneidade das variâncias dos tratamentos e Fischer para Análise de Variância, foram feitas através do programa Assistat 7.7 Beta, e as médias comparadas pelo teste de Tukey a 5\% de probabilidade.

\section{RESULTADOS E DISCUSSÃO}

Os parâmetros altura (h) e diâmetro do coleto (DC), relacionados ao desenvolvimento morfológico das mudas de Dipteryx odorata sob diferentes dosagens de adubação fosfatada, apresentaram-se estatisticamente semelhantes de acordo com o teste de Tukey a 5\% de probabilidade, assim como as variáveis de área foliar (AF) e índice de qualidade de mudas (IQD) (Tabela 1).

Segundo Birchler et al. (1998) e Hunt (1990), o valor de IQD deve ser maior que 0,2 , mas alguns estudos mostram que O IQD é um parâmetro variável, diferenciando de acordo com a espécie, manejo das mudas em viveiro, tipo e proporção do substrato, volume do recipiente, idade, níveis e tipo de adubação, entre outros (GASPARIN, 2012).

Neste sentido, conforme os dados deste trabalho, as mudas provenientes de todos os tratamentos a níveis de adubação a base de fosforo (P), apresentam valores que permitem sua instalação em campo, mesma as advindas do tratamento $2(200 \mathrm{Kg} / \mathrm{ha}$ de P) que apresentou menor IQD $(1,74)$, juntamente com 0 tratamento testemunha $(1,68)$. 
Tabela 1. Análise de variância e teste de médias para os parâmetros morfológicos altura (h), diâmetro do coleto $(\varnothing)$, área foliar (AF), quantidade de folhas (QF)e Índice de Qualidade de Dickson (IQD).

\begin{tabular}{ccccc}
\hline Tratamentos & $\mathrm{h}(\mathrm{cm})$ & $\varnothing(\mathrm{mm})$ & $\mathrm{AF}\left(\mathrm{mm}^{2}\right)$ & IQD \\
\hline 0 & $11,07 \mathrm{a}$ & $0,2350 \mathrm{a}$ & $7015,21 \mathrm{a}$ & $1,6788 \mathrm{a}$ \\
1 & $17,25 \mathrm{a}$ & $0,4175 \mathrm{a}$ & $7563,38 \mathrm{a}$ & $4,0420 \mathrm{a}$ \\
2 & $14,75 \mathrm{a}$ & $0,3050 \mathrm{a}$ & $4341,55 \mathrm{a}$ & $1,7368 \mathrm{a}$ \\
3 & $15,75 \mathrm{a}$ & $0,3650 \mathrm{a}$ & $9432,66 \mathrm{a}$ & $3,4594 \mathrm{a}$ \\
4 & $13,50 \mathrm{a}$ & $0,3825 \mathrm{a}$ & $9113,61 \mathrm{a}$ & $2,8754 \mathrm{a}$ \\
\hline ANOVA & $\mathrm{h}(\mathrm{cm})$ & $\varnothing(\mathrm{mm})$ & $\mathrm{AF}\left(\mathrm{mm}^{2}\right)$ & IQD \\
\hline QM & $21,9100^{\mathrm{ns}}$ & $0,2068^{\mathrm{ns}}$ & $16553505,5655^{\mathrm{ns}}$ & $4,3620^{\mathrm{ns}}$ \\
p-valor & 0,2183 & 0,0821 & 0,1567 & 0,0321 \\
CV\% & 25,35 & 26,39 & 39,03 & 40,32 \\
Média & 9,50 & 0,2250 & 7029,88 & 2,46 \\
Shapiro-Wilk (W) & 0,68675 & 0,79268 & 0,9460 & 0,9338 \\
p-valor & 0,00003 & 0,00067 & 0,3106 & 0,1833 \\
\hline
\end{tabular}

** Significativo ao nível de $1 \%$ de probabilidade $(p<0.01)$; Significativo ao nível de $5 \%$ de probabilidade $(0.01=<p<0.05)$; ns Não Significativo $(p>=0.05)$. As médias seguidas pela mesma letra não diferem estatisticamente entre si. Foi aplicado o Teste de Tukey ao nível de $5 \%$ de probabilidade e o Teste de Normalidade de Shapiro-Wilk (W).

De acordo com Munguambe (2013), a altura é uma das mais importantes variáveis mensuradas nas plantas, pois se pode selecionar e classificar mudas, além de ser uma característica de fácil avaliação. Por esta razão, através dos dados obtidos do parâmetro altura foi possível observar se os tratamentos utilizados surtiram efeitos no desenvolvimento vertical das plântulas e consequentemente sobre o acúmulo de biomassa e qualidade das mudas.

Oliveira et al. (2008), mostram que plantas com maior diâmetro, apresentam maior sobrevivência, por apresentarem capacidade de formação e de crescimento de novas raízes.

No entanto, o Teste de Tukey não acusou diferenças significativas para altura e diâmetro de planta, mostrando semelhança nos efeitos da adubação em diferentes níveis de $\mathrm{P}$, dados que refletem diretamente no incremento de área foliar (AF) e no índice de qualidade das plântulas (IQD), que também mostraram-se estatisticamente iguais de acordo com o teste de médias usado (Tabela 1). 
Na Figura 1 pode ser observado o crescimento médio em altura das mudas de Dipteryx odorata durante as quatro avaliações após a adubação fosfatada.

Figura 1. Crescimento médio em altura das mudas de Dipteryx odorata.

\section{Curva de Crescimento}

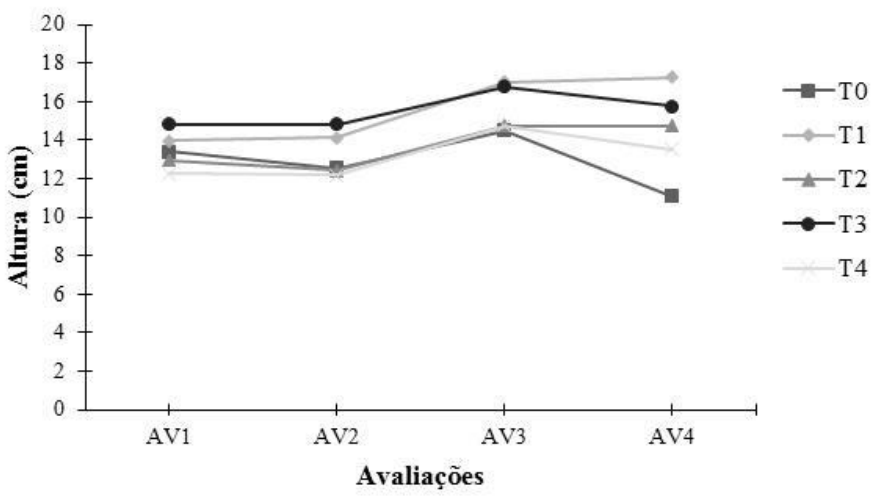

Observa-se que a partir da curva, as mudas comportaram-se de forma semelhante sob o efeito da adubação fosfatada durante as três primeiras avaliações, no entanto, na quarta avaliação nota-se que apenas os tratamentos 1 (100 Kg/ha de P) e 2 (200Kg/ha de P) apresentam tendência à desenvolvimento, enquanto que os demais indicam redução de biomassa, provavelmente em decorrência da superadubação, que acarretou carência em incremento das plântulas durante o desenvolvimento inicial.
Em estudo semelhante, ao avaliar o crescimento e a qualidade de mudas de Schizolobium parahyba, Garcia e Souza (2015) utilizando adubo fosfato conseguiram mudas com altura média de 21,98cm, diâmetro de 0,79 mm e IQD de 1,26 aos 96 dias de idade. Dados próximos aos obtidos no presente estudo, que indicou a melhor média de altura em 17,25 cm, diâmetro 0,41 mm e IQD 4,04 aos 60 dias de avaliações.

O parâmetro acúmulo de biomassa após análise de variância apontou 
diferenças significativas para os acusando não significância para o parâmetros massa seca aérea (MSA) e parâmetro (MSR), conforme disposto massa seca total (MST) estudados e na (Tabela 2).

Tabela 2. Análise de variância (ANAVA) para acúmulo de biomassa das matrizes estudadas.

\begin{tabular}{cccc}
\hline Tratamentos & MAS & MSR & MST \\
\hline 0 & $0,77 \mathrm{c}$ & $0,34 \mathrm{a}$ & $1,11 \mathrm{c}$ \\
1 & $2,78 \mathrm{a}$ & $0,71 \mathrm{a}$ & $3,49 \mathrm{a}$ \\
2 & $0,91 \mathrm{bc}$ & $0,48 \mathrm{a}$ & $1,39 \mathrm{bc}$ \\
3 & $2,17 \mathrm{ab}$ & $0,68 \mathrm{a}$ & $2,85 \mathrm{ab}$ \\
4 & $1,71 \mathrm{abc}$ & $0,62 \mathrm{a}$ & $2,33 \mathrm{abc}$ \\
\hline & MAS & MSR & MST \\
\hline QM & $2,8756^{\star \star}$ & $0,0972 \mathrm{~ns}$ & $3,9576^{\star \star}$ \\
p-valor & 0,0012 & 0,1108 & 0,0025 \\
CV\% & 36,26 & 36,77 & 34,31 \\
Média & 1,43 & 0,52 & 1,79 \\
Shapiro-Wilk (W) & 0,9088 & 0,9635 & 0,9078 \\
p-valor & 0,0606 & 0,6164 & 0,0580 \\
\hline
\end{tabular}

** Significativo ao nível de $1 \%$ de probabilidade $(p<0.01)$; * Significativo ao nível de $5 \%$ de probabilidade $(0.01=<p<0.05)$; ns Não Significativo ( $p>=0.05)$. As médias seguidas pela mesma letra não diferem estatisticamente entre si. Foi aplicado o Teste de Tukey ao nível de 5\% de probabilidade e o Teste de Normalidade de Shapiro-Wilk (W).

Verificou-se que diante dos Munguambe (2013) esse tipo de diferentes tratamentos à base de doses comportamento é característico das de $P$, as plântulas apresentaram plantas, pois as raízes estão semelhante nível de desenvolvimento intimamente associadas as atividades em biomassa da parte radicular (MSR), de natureza fisiológica das mudas, no mesmo o incremento em biomassa da complexo ambiente-solo-água-planta, parte aérea (MSA) apresentando-se na qual são importantes para o estatisticamente diferentes entre os funcionamento dos processos tratamentos usados. Segundo fisiológicos e desenvolvimento. A parte 
aérea também tem grande importância na qualidade das mudas, uma vez que é responsável por fornecer nutrientes, fitormônios e carboidratos para as raízes, e esta por sua vez, fornece água e outros nutrientes para a parte aérea, formando uma relação fundamental para o desenvolvimento da planta.

Observa-se que nos parâmetros morfológicos estudados, o tratamento 1 (adubação de 100 Kg/ha de P), indicou maiores pesos de massa seca aérea (MSA), $(2,78)$ e massa seca total (MST), (3,49 g), enquanto que o tratamento testemunha e o tratamento 2 ( $200 \mathrm{~kg} /$ ha de P) apresentaram os mais inferiores valores de acúmulo de biomassa, com MAS e MST de 0,77g ; $1,11 \mathrm{~g}$ e $0,91 \mathrm{~g} ; 1,39$, respectivamente. 0 acúmulo de massa nas plantas tem sido considerado um dos melhores parâmetros para caracterizar a qualidade de mudas e seu comportamento em campo, por esse motivo seu estudo é de fundamental importância e no presente trabalho o tratamento 1 mostrou-se fisiologicamente superior, indicando melhores efeitos sobre o desenvolvimento inicial de mudas de Dipteryx odorata.

\section{CONCLUSÃO}

A dosagem a nível de $200 \mathrm{Kg} / \mathrm{ha}$ de P, configura-se como o tratamento mais significativo ao desenvolvimento de mudas de Dipteryx odorata em viveiro.

Os parâmetros de desenvolvimento morfológico das plântulas: altura; diâmetro e área foliar, apresentaramse não significativo à todos os tratamentos à base de adubação fosfatada adotados.

O Índice de qualidade de mudas calculado (IQD) indicou que todos os níveis de adubação fosfatada testados, geraram mudas aptas a instalação em campo, após 60 dias em condições de viveiro.

\section{REFERÊNCIAS}

CECONI, D. E.; POLETTO, I.; BRUN, E. J.; LOVATO, T. Crescimento de mudas de Açoita-cavalo (Luehea divaricata Mart.) sob influência da adubação fosfatada. 
Cerne, Lavras, v. 12, n. 3, p. 292-299, 2006.

DICKSON, A.; LEAF, A. L.; HOSNER, J. F. Quality appraisal of white spruce and white pine seedling stock in nurseries. Forestry Chronicle, v. 36, p. 10-13, 1960.

GARCIA, E. A.; SOUZA, J. P. Avaliação da qualidade de mudas de Schizolobium parahyba em função de diferentes aplicações de adubo fosfatado. Tekhne e Logos. Botucatu, v. 6, n. 1, pag. 51-59, 2015.

GASPARIN, E. Armazenamento de sementes e produção de mudas de Parapiptadenia rígida (Benth.) Brenan. 2012. 146 f. Dissertação (Mestrado em Engenharia Florestal) - Universidade Federal de Santa Maria - UFSM, Santa Maria, 2012.

HUNT, G. A. Effect of styroblock design and cooper treatment on morphology of conifer seedlings. In: TARGET SEEDLINGS SYMPOSIUM, MEETING OF THE WESTERN FOREST NURSERY ASSOCIATIONS, GENERAL TECHNICAL REPORT RM-200, 1., 1990, Roseburg. Proceedings... Fort Collins: USDA. 1990.

LORENZI, H. Árvores Brasileiras: Manual de identificação e Cultivo de Plantas Arbóreas Nativas do Brasil. 1 ed. Editora Nova Odessa. São Paulo: Instituto Plantarum. Vol. 2. 1998.

MOREIRA, J. F.; BARROS, N. F.; NOVAIS, R. F. Efeito do tempo de contato do fósforo com o solo sobre sua disponibilidade para mudas de eucalipto. Revista Brasileira de Ciência do Solo, Campinas, v. 15, p. 303-308, 1991.

MUNGUAMBE, J. F. Qualidade morfológica de mudas clonais de eucalipto na fase de expedição em viveiros comerciais. 2013. 74 p. Dissertação (Mestrado em Ciência Florestal) - Universidade Federal Lavras - UFL, Lavras, 2013.

OLIVEIRA, R. B.; LIMA, J. S. S.; SOUZA, C. A. M.; SILVA, S. A.; FILHO, S. M. Produção de mudas de essências florestais em diferentes substratos e acompanhamento

do desenvolvimento em campo. Ciência Agrotécnica. Lavras, v. 32, n. 1, p. 122128, 2008.

ZUCAREL, C.; RAMOS JUNIOR, E. U.; BARREIRO, A. P.; NAKAGAWA, J.; CAVARIANI, C. Adubação fosfatada, componentes de produção, produtividade e qualidade fisiológica em sementes de feijão. Revista Brasileira de Sementes, v. 28, n. 1, p. 915, 2006. 